\title{
MYBL2 in synergy with CDC20 promotes the proliferation and inhibits apoptosis of gastric cancer cells
}

\author{
Qianxi Deng ${ }^{1, A, F}$, Linju Wu ${ }^{2, B, D}$, Yiming $\mathrm{Li}^{1, C, E}$, Long Zou ${ }^{1, B, E}$ \\ ${ }^{1}$ Department of Gastroenterology, The Third Hospital of Mianyang, Sichuan Mental Health Center, China \\ ${ }^{2}$ Department of Anesthesiology, The Third Hospital of Mianyang, Sichuan Mental Health Center, China \\ A - research concept and design; $B$ - collection and/or assembly of data; $C$ - data analysis and interpretation; \\ $D$ - writing the article; $E$ - critical revision of the article; $F$ - final approval of the article
}

\section{Address for correspondence \\ Qianxi Deng}

E-mail: dengqianxi12@163.com

Funding sources

None declared

Conflict of interest

None declared

Received on December 30, 2020

Reviewed on March 9, 2021

Accepted on April 20, 2021

Published online on August 6, 2021

Cite as

Deng $Q$, Wu L, Li Y, Zou L. MYBL2 in synergy with CDC20

promotes the proliferation and inhibits apoptosis of gastric

cancer cells. Adv Clin Exp Med. 2021;30(9):957-966.

doi:10.17219/acem/135938

DOI

10.17219/acem/135938

\section{Copyright}

Copyright by Author(s)

This is an article distributed under the terms of the

Creative Commons Attribution 3.0 Unported (CC BY 3.0)

(https://creativecommons.org/licenses/by/3.0/)

\begin{abstract}
Background. Gastric cancer (GC) is a common malignant tumor with a high morbidity and mortality worldwide. It has been reported that V-Myb avian myeloblastosis viral oncogene homolog-like 2 (MYBL2) could be a promising prognostic biomarker for GC. However, the specific role of MYBL2 in GC progression remains unclear.
\end{abstract}

Objectives. To examine the role of MYBL2 in GC progression and investigate the underlying mechanisms.

Materials and methods. The mRNA levels of target genes were detected using quantitative real-time polymerase chain reaction (RT-qPCR) and protein expression was measured with western blot analysis. Cell Counting Kit-8 (CCK-8) and colony formation assays were employed to inspect HGC-27 cell proliferation, and cellular apoptosis was determined with TUNEL staining. Finally, the interaction of MYBL2 and cell division cycle 20 (CDC20) was verified with immunoprecipitation.

Results. MYBL2 was confirmed to be overexpressed in GC cells. MYBL2 knockdown inhibited HGC-27 cell proliferation and promoted cellular apoptosis, and these effects were reversed by CDC20 overexpression. Interestingly, MYBL2 interacted with CDC20 and regulated its expression. MYBL2 knockdown also inhibited activation of the Wnt/B-catenin signaling pathway, while CDC20 overexpression showed the opposite effect.

Conclusions. In summary, the synergy between MYBL2 and CDC20 induced the proliferation of GC cells and inhibited cell apoptosis; these effects may have involved the Wnt/B-catenin signaling pathway. Thus, MYBL2 may be a promising target for $\mathrm{GC}$ treatment.

Key words: apoptosis, gastric cancer, proliferation, cell division cycle 20 (CDC20), V-Myb avian myeloblastosis viral oncogene homolog-like 2 (MYBL2) 


\section{Background}

Gastric cancer (GC) is the $5^{\text {th }}$ most common malignant tumor and the associated mortality rate is $3^{\text {rd }}$ globally. ${ }^{1,2}$ There are more than one million new GC cases diagnosed worldwide each year and this disease has become a major burden on human health. ${ }^{3}$ Since patients with early GC have no obvious symptoms, the majority are already at an advanced stage at the time of diagnosis. ${ }^{4,5}$ While great progress has been achieved through the development of new diagnostic methods and treatment strategies, the five-year survival rate in advanced patients has not exceeded $15 \% .{ }^{6}$ Due to the complex molecular mechanisms involved in the onset and development of $\mathrm{GC}$, the specifics of $\mathrm{GC}$ pathogenesis are still unclear. Hence, it is important to explore novel and effective biomarkers for the diagnosis, treatment and prognosis of GC.

$\mathrm{V}-\mathrm{Myb}$ avian myeloblastosis viral oncogene homologlike 2 (MYBL2) is a member of the MYB family of transcription factors and is involved in the regulation of infinite replicative potential, evasion from apoptosis, tissue infiltration, and metastasis. ${ }^{7}$ Expression of $M Y B L 2$ is ubiquitous and can be observed in almost every proliferating cell. ${ }^{8}$ Overexpression of this gene is also associated with a poor prognosis in multiple cancers. ${ }^{7}$ Upregulation of $M Y B L 2$, a key downstream effect of Akt/FoxM1 signaling, facilitates the progression of glioma. ${ }^{9}$ Recently, MYBL2 overexpression has been also observed in malignant tumors including colorectal cancer, ${ }^{10}$ acute myeloid leukemia ${ }^{11}$ and breast cancer, ${ }^{12}$ suggesting that this gene plays an essential role in tumor cell growth and carcinogenesis. A search of the CCLE database (https://portals.broadinstitute.org/ ccle) suggests that $M Y B L 2$ is generally upregulated in numerous tumor cell lines. Interestingly, it has been reported that MYBL2 is relevant to cancer cell differentiation and lymph node metastasis. Studies have shown that its expression is negatively correlated with the survival rate of GC patients, suggesting that this gene could be a promising prognostic biomarker for gastric adenocarcinoma. ${ }^{6}$ However, the specific role of $M Y B L 2$ in the occurrence and progression of $\mathrm{GC}$ remains unclear.

Cell division cycle 20 (CDC20), a gene first discovered in yeast, plays an essential role in the progress of cell cycle. ${ }^{13}$ CDC20 is an indispensable developmental gene as its inhibition in mice leads to chromosome condensation and embryonic death, partly due to abnormal mitosis. ${ }^{14} C D C 20$ ablation can also effectively inhibit the invasiveness of mouse skin tumors, mainly due to increased apoptosis. ${ }^{15}$ In addition, a retrospective study identified $C D C 20$ expression as a useful biomarker for the prognosis of pancreatic cancer. ${ }^{16}$ This gene has also been studied in a great diversity of other tumors. ${ }^{17}$

\section{Objectives}

The results cited above suggest that $M Y B L 2$ is very promising as a biomarker for the prognosis of GC. Thus, the aim of this study is to verify the role of $M Y B L 2$ in GC progression, and more importantly, to investigate the underlying mechanisms.

\section{Materials and methods}

\section{Cell culture and transfection}

Human GC cell lines, including MKN-45, MKN-74, AGS and HGC-27, and a normal gastric GES-1 cell line were purchased from the Type Culture Collection of the Chinese Academy of Sciences (Shanghai, China). All of these cell lines were cultured in RPMI-1640 medium (Gibco, Thermo Fisher Scientific, Waltham, USA) containing 10\% fetal bovine serum (FBS; Thermo Fisher Scientific), $100 \mathrm{U} / \mathrm{mL}$ of penicillin-G, and $100 \mu \mathrm{g} / \mathrm{mL}$ of streptomycin. The cells were maintained in a $37^{\circ} \mathrm{C}$ humidified atmosphere with $5 \% \mathrm{CO}_{2}$.

Small interfering (si)-MYBL2 (si-MYBL2-1 5'-CCAAGAGCACACCTGTTAA-3'; si-MYBL2-2 5'-CCAGAAACATGCTGCGTTT-3'), and the scramble siRNA (si-NC, 5'-ACGTGACACGTTCGGAGAATT-3') as a negative control (NC), were obtained from Shanghai GenePharma Co., Ltd. (Shanghai, China). The si-MYBL2 (50 nM) and si-NC $(50 \mathrm{nM})$ were transfected into HGC-27 cells $\left(5 \times 10^{5}\right.$ cells/ well) using Lipofectamine 2000 (Invitrogen, Carlsbad, USA) according to the manufacturer's instructions. In addition, CDC20 transcript cDNA was inserted into the pCDNA3.1 by Lederer Biological Technology (Guangdong, China), and then transfected into HGC-27 cells $(20 \mu \mathrm{g})$ to achieve CDC20 overexpression (Ov-CDC20). An empty vector without $C D C 20$ sequence was used as the negative control (OV-NC).

\section{RT-qPCR}

Total RNA from HGC-27 cells was extracted using TRIzol ${ }^{\circledR}$ reagent (Invitrogen; Thermo Fisher Scientific) following the manufacturer's protocol. Complementary DNA (cDNA) was synthesized using a Reverse Transcription kit (Thermo Fisher Scientific) according to manufacturer's instructions. Real-time quantitative polymerase chain reaction (RT-qPCR) was performed using Roche SYBR Green PCR kits (Roche Diagnostics, Basel, Switzerland) and carried out using the Opticon Real-Time PCR Detection System (ABI 7500; Life Technologies, Carlsbad, USA). The GAPDH gene was used as an internal gene for normalization. The cycling conditions were as follows: 1 cycle of $95^{\circ} \mathrm{C}$ for $2 \mathrm{~min}$ and 40 cycles of $95^{\circ} \mathrm{C}$ for $15 \mathrm{~s}$, with a final extension at $60^{\circ} \mathrm{C}$ for $60 \mathrm{~s}$. The relative mRNA quantity was calculated using the $2^{-\Delta \Delta \mathrm{Cq}}$ method. ${ }^{18}$ The primer sequences were as follows: MYBL2 forward, 5'AAAACAGTGAGGAGGAAC-3' and reverse, 5'CAGGGAGGTCAAATTTAC-3'; CDC20 forward, 5'-GGCACCAGTGATCGACACATTCGCAT-3' and reverse, 5'-GCCATAGCCTCAGGGTCTCATCTGCT-3'; and GAPDH forward, 5'CTGGGCTACACTGAGCACC-3' and reverse, 5'AAGTGGTCGTTGAGGGCAATG-3'. 


\section{Western blot analysis}

The cells were washed with cold phosphate-buffered saline (PBS) and then lysed in a lysis buffer supplemented with phenylmethylsulfonyl fluoride $(1 \mathrm{mM})$, trypsin $(10 \mu \mathrm{g} / \mathrm{mL})$, aprotinin $(10 \mu \mathrm{g} / \mathrm{mL})$, and leupeptin $(10 \mu \mathrm{g} / \mathrm{mL})$. A bicinchoninic acid (BCA) protein assay was used to quantify the protein concentration. Proteins $(25 \mu \mathrm{g} /$ lane $)$ were separated in a $10 \%$ sodium dodecyl sulphate-polyacrylamide gel electrophoresis (SDS-PAGE) gel and then transferred onto polyvinylidene difluoride PVDF membranes. The membranes were then blocked with skim milk for $2 \mathrm{~h}$ at room temperature and subsequently incubated with primary antibodies against MYBL2 (\#PA5-79713; $1: 1000)$, PCNA (\#133900; 1:1000), Ki-67 (\#14-5698-82; 1: 1000), Bcl-2 (\#MA511757; 1: 1000), Bax (\#33-6400; 1:1000), cleaved caspase-3 (\#ab2302; 1:500; Abcam, Cambridge, UK), caspase-3 (\#MA5-11521; 1:1000), CDC20 (\#PA5-63103; 1:1000), cMyc (\#MA1-980; $1: 1000), \beta$-catenin (\#MA1-301; $1: 1000$ ), p-GSK-3 $\beta$ (\#MA5-14873; $1: 500)$ ) and GSK-3 $\beta$ (\#39-9500; $1: 1000)$ at $37^{\circ} \mathrm{C}$ overnight. GAPDH (\#39-8600; $\left.1: 1000\right)$ was used as a loading control. After washing with PBS 3 times, the polyvinylidene difluoride (PVDF) membranes were incubated with horseradish peroxidase (HRP)-goat anti-rabbit secondary antibody (\#G-21234; 1:50000; Invitrogen) for $2 \mathrm{~h}$ at room temperature, and the intensities of the bands were analyzed using ImageJ software v. 1.6 (National Institutes of Health, Bethesda, USA). Antibodies that are not branded were obtained from Thermo Fisher Scientific.

\section{CCK-8 assay}

A Cell Counting Kit-8 (CCK-8) assay (Beyotime Institute of Biotechnology, Haimen, China) was used to investigate the effects of $M Y B L 2$ knockdown and $C D C 20$ overexpression on the viability of HGC-27 cells. Cells were seeded in 3 independent 96 -well plates $\left(5 \times 10^{3}\right.$ cells/well $)$ and incubated for 24 and $48 \mathrm{~h}$ at $37^{\circ} \mathrm{C}$. Following this, CCK- 8 reagent $(10 \mu \mathrm{L})$ was added into each well and the plates were subsequently incubated at $37^{\circ} \mathrm{C}$ for another $2 \mathrm{~h}$. The absorbance at $450 \mathrm{~nm}$ was detected using an enzymelinked immunosorbent assay (ELISA) plate reader (BioRad, Hercules, USA).

\section{Colony formation assay}

The colony formation assay was performed to detect the effect of $M Y B L 2$ knockdown and $C D C 20$ overexpression on cell proliferation. After transfection, cells $\left(1 \times 10^{3} /\right.$ well $)$ were seeded in a $35-\mathrm{mm}$ petri dish and incubated for 10 days at $37^{\circ} \mathrm{C}$ to form colonies. Subsequently, HGC-27 cells were fixed with $4 \%$ paraformaldehyde for $5 \mathrm{~min}$ and stained with $0.1 \%$ crystal violet solution for $20 \mathrm{~min}$ at room temperature. The number of colonies (diameters $>0.5 \mathrm{~mm}$ ) within a field was counted using a digital camera (Nikon Corp., Tokyo, Japan).

\section{TUNEL staining}

HGC-27 cells $\left(1 \times 10^{5}\right.$ cells $\left./ \mathrm{mL}\right)$ were seeded in sixwell plates and then fixed in $4 \%$ paraformaldehyde for 5 min at room temperature. After permeabilization with 0.1\% Triton X-100 (Sigma-Aldrich, Merck KGaA, St. Louis, USA) for $5 \mathrm{~min}, \mathrm{HGC}-27$ cells were stained according to the protocol of the ApopTag Fluorescein In Situ Apoptosis Detection kit (Chemicon International Inc., Temecula, USA). Nuclei were labeled with 4',6-diamidino-2-phenylindole (DAPI) and quantified under a fluorescence microscope at $\times 200$ magnification (Leica Microsystems GmbH, Wetzlar, Germany).

\section{Immunoprecipitation}

Cells were collected and lysed with immunoprecipitation (IP) lysis buffer containing protease inhibitors. After centrifugation at $12,000 \times \mathrm{g}$ at $4^{\circ} \mathrm{C}, M Y B L 2$ antibody $(1 \mu \mathrm{g})$ was added into the supernatant and the samples were placed on a rotating platform overnight at $4^{\circ} \mathrm{C}$. Subsequently, $50 \mu \mathrm{L}$ of SureBeads ${ }^{\mathrm{TM}}$ protein $\mathrm{G}$ magnetic beads (No. 1614023; Bio-Rad) were added into the above mixture at $4^{\circ} \mathrm{C}$ with gentle rotation for $4 \mathrm{~h}$. The pellets were dissolved in $60 \mu \mathrm{L} \times 1$ electrophoresis sample buffer and boiled for $5 \mathrm{~min}$. Samples $(30 \mu \mathrm{L})$ were analyzed using western blot analysis as outlined above.

\section{Statistical analyses}

Data are presented as the mean \pm standard error of mean (SEM) of at least 3 experiments. Statistical analyses was performed using SPSS v. 17.0 software (SPSS Inc., Chicago, USA). Analysis of variance (ANOVA) followed by Bonferroni's post hoc test were used to determine the differences in the means among multiple groups. P-value $<0.05$ was considered to indicate a statistically significant difference.

\section{Results}

\section{MYBL2 is highly expressed in GC cells}

A search of the CCLE database suggested that MYBL2 is generally upregulated in numerous tumor cell lines, including GC cell lines (Fig. 1A). To explore the effect of $M Y B L 2$ on GC progression, the mRNA and protein expression of $M Y B L 2$ were detected using RT-qPCR and western blot analysis, respectively. The mRNA and protein expression of $M Y B L 2$ is significantly upregulated in GC cell lines, including MKN-45, MKN-74, AGS, and HGC-27, compared to the GES-1 cell line, suggesting that MYBL2 may play an oncogenic role in the onset and development of GC (Fig. 1B,C). 


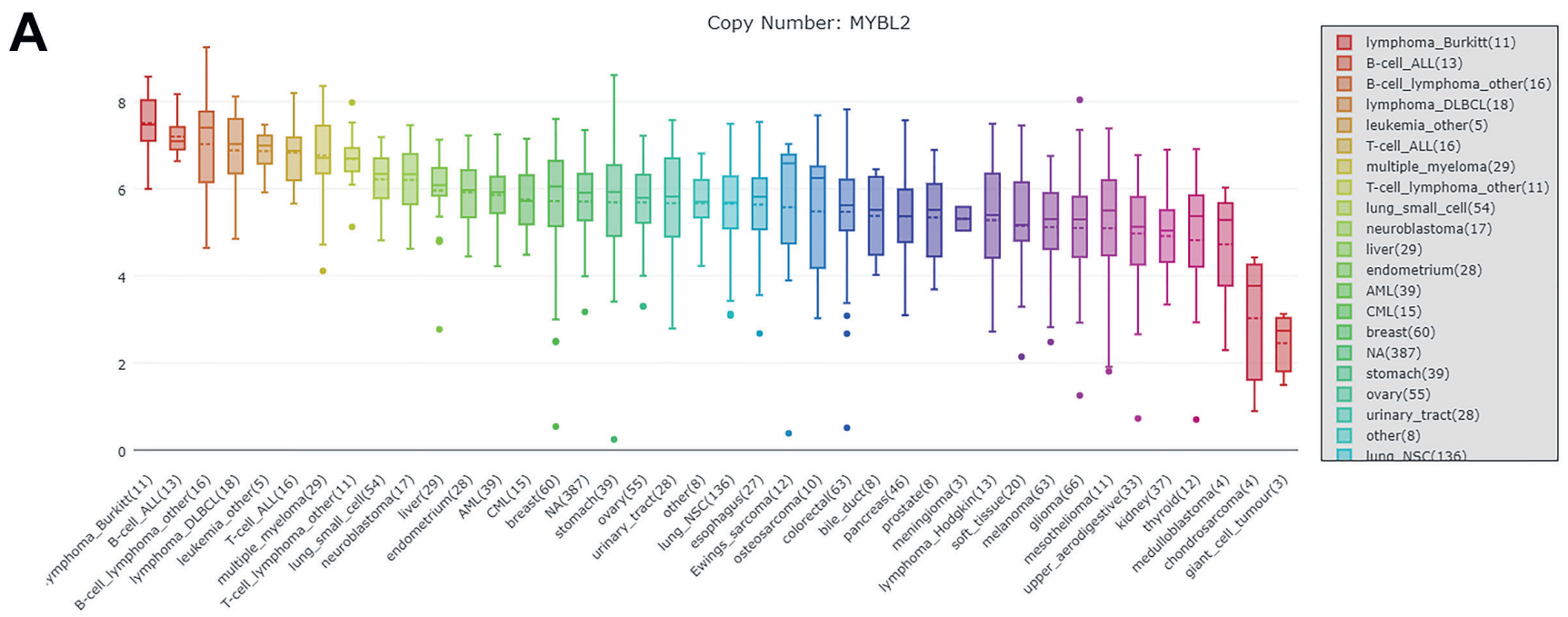

B

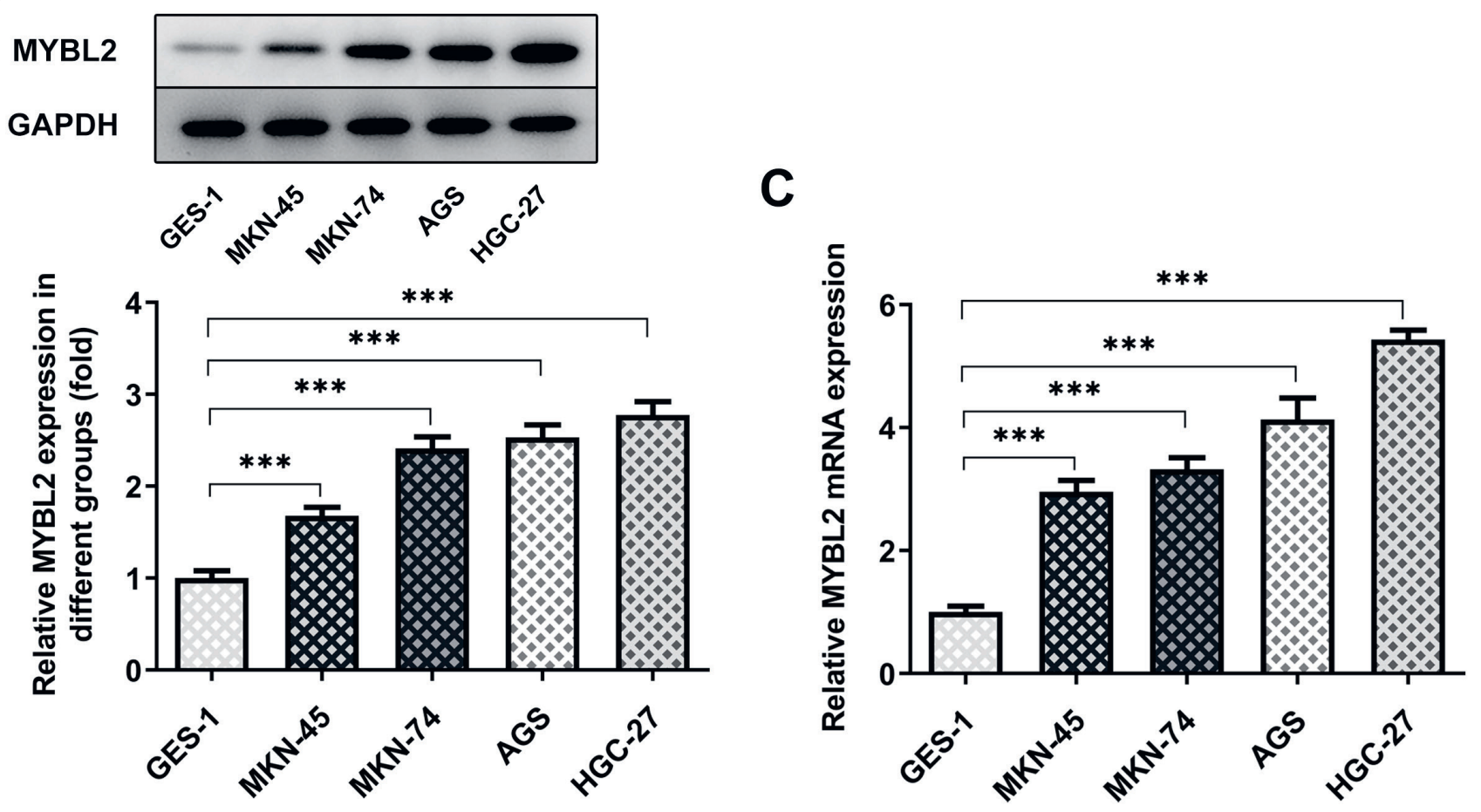

Fig. 1. MYBL2 is highly expressed in gastric cancer (GC) cells. A. The MYBL2 expression in multiple cancer cell lines in the CCLE database; B. The MYBL2 protein expression was determined with western blot analysis and quantification; C. The MYBL2 mRNA level was analyzed with quantitative real-time polymerase chain reaction (RT-qPCR). Error bars represent the mean \pm standard error of mean (SEM) from 3 independent experiments

\section{MYBL2 silencing inhibits HGC-27 cell growth}

To examine the specific effect of MYBL2 on GC progression, cell growth was analyzed with CCK-8 and colony formation assays. The si-MYBL2 was used to achieve $M Y B L 2$ knockdown. As shown in Fig. 2A, si-MYBL2 caused a significant reduction in the MYBL2 mRNA level, especially in the si-MYBL2-1 group. Hence, si-MYBL2-1 was selected for the subsequent experiments. Moreover, the results of the CCK- 8 assay showed that viability was significantly depressed by MYBL2 knockdown at the indicated time ( $24 \mathrm{~h}$ and $48 \mathrm{~h}$ ), as compared to the control (Fig. 2B). The results of the western blot analysis demonstrated that the expression levels of proliferative markers, including proliferating cell nuclear antigen (PCNA) and Ki-67, were decreased in HGC-27 cells from the si-MYBL2 group (Fig. 2C,D). Finally, the results of the colony formation assay showed that MYBL2 silencing remarkably suppressed the proliferation of HGC-27 cells (Fig. 2E). These results indicate that MYBL2 silencing inhibits HGC-27 cell growth. 

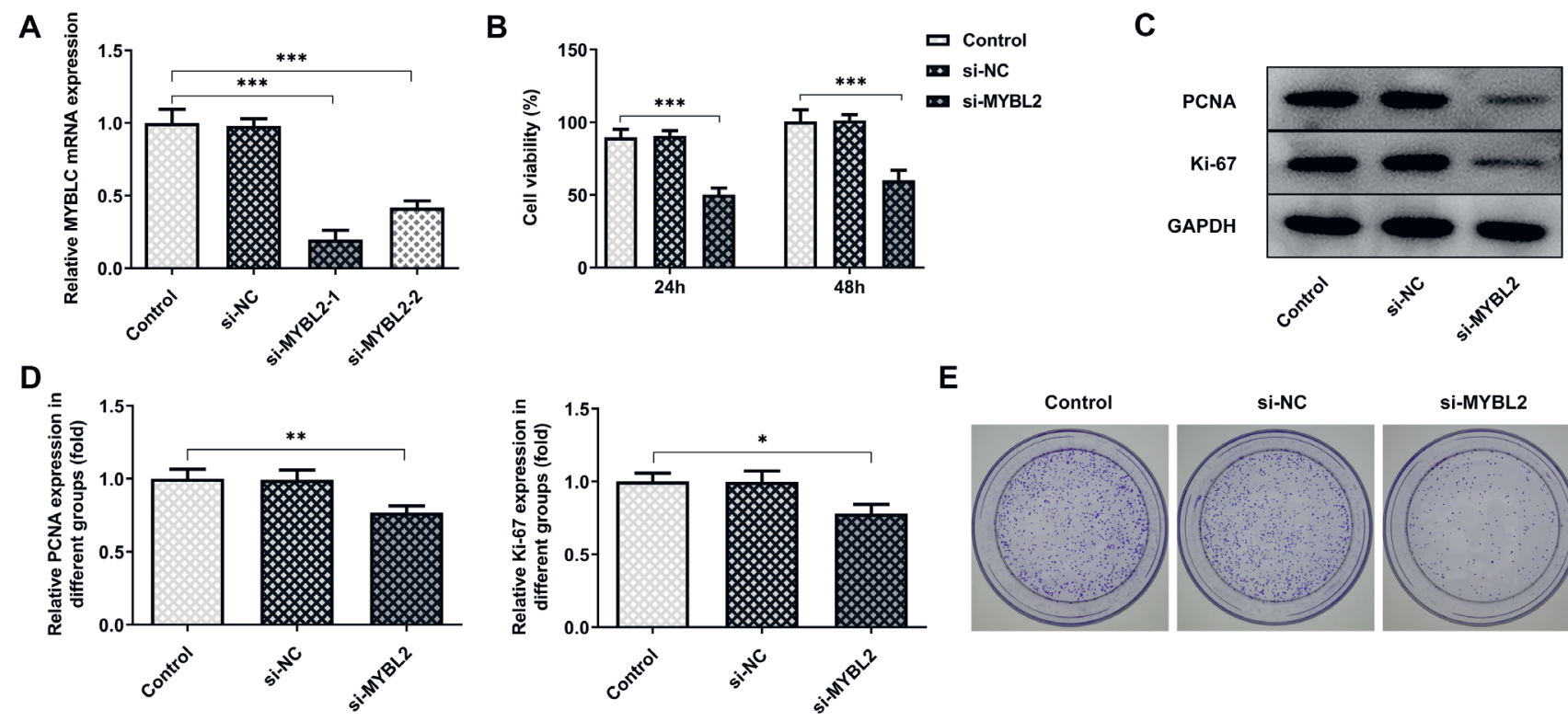

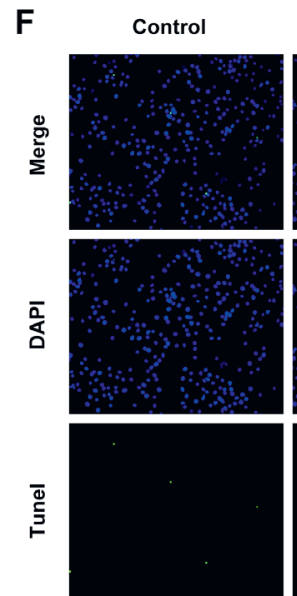

I

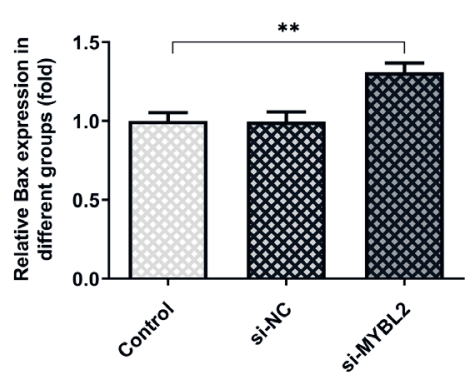

si-NC
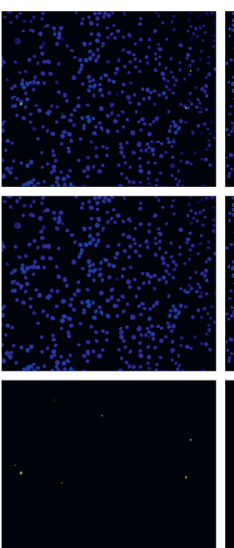

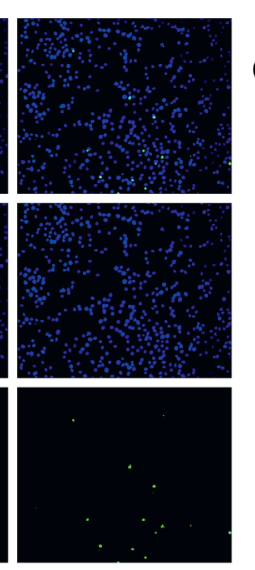

G

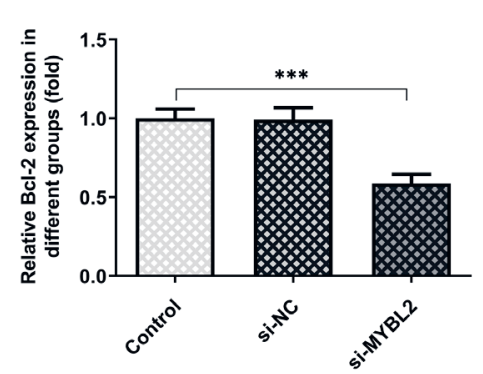

E
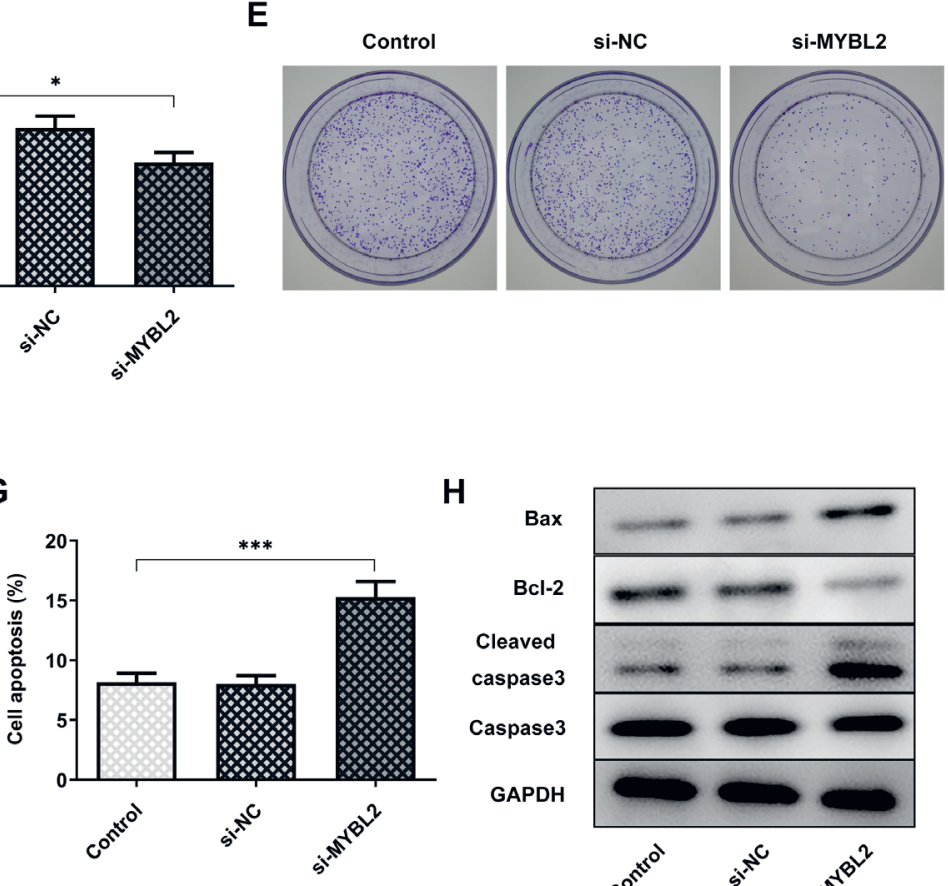

H

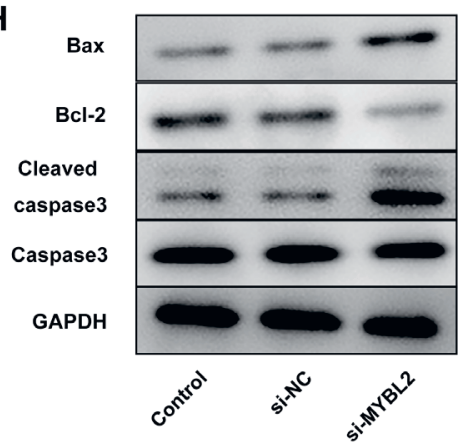

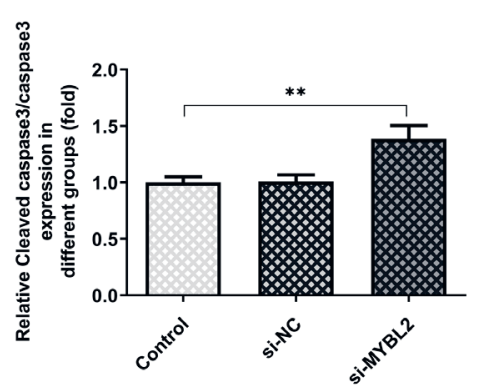

Fig. 2. MYBL2 silencing inhibits cells growth and promotes cell apoptosis of HGC-27 cells. A. The MYBL2 mRNA level was analyzed with quantitative realtime polymerase chain reaction (RT-qPCR); B. The HGC-27 cell viability was detected using the Cell Counting Kit-8 (CCK-8) assay; C and D. The proliferating cell nuclear antigen (PCNA) and Ki-67 protein expressions were determined with western blot analysis and quantification; E. HGC-27 cell proliferation was analyzed using colony formation assay; F and G. The HGC-27 cell apoptosis was determined with TUNEL staining; H and I. Bax, Bcl-2, cleaved caspase-3, and caspase-3 protein expressions were determined using western blot analysis and quantification. Error bars represent the mean \pm standard error of mean (SEM) from 3 independent experiments

${ }^{*} p<0.05 ;{ }^{* *} p<0.01 ;{ }^{* * *} p<0.001$.

\section{MYBL2 silencing promotes} the apoptosis of HGC-27 cells

Apoptosis was analyzed by TUNEL staining to detect the specific effect of $M Y B L 2$ on GC progression. The HGC-27 cell apoptosis rate in the si-MYBL2-1 group was higher than that in the control group (Fig. 2F,G).
Additionally, the expression levels of Bax (pro-apoptotic), Bcl-2 (anti-apoptotic) and cleaved caspase-3/caspase-3 were assessed using western blot analysis. MYBL2 silencing led to the loss of $\mathrm{Bcl}-2$ and upregulation of $\mathrm{Bax}$ and cleaved caspase-3 (Fig. 2H,I). These results imply that MYBL2 silencing promotes the apoptosis of HGC-27 cells. 


\section{MYBL2 interacts with CDC20 and regulates its expression}

To investigate the underlying mechanism of $M Y B L 2$ in GC progression, studies were carried out to explore its downstream targets. Based on the LinkedOmics website (www.linkedomics.org), the correlation between the $M Y B L 2$ and $C D C 20$ in GC was analyzed. It was found that $M Y B L 2$ expression is positively correlated with most genes (Fig. 3A,B), and is highly correlated with $C D C 20$ (p < 0.0001; Fig. 3C). Furthermore, the coexpression of $M Y B L 2$ and $C D C 20$ in the Gene Expression Omnibus (GEO) database was analyzed using COEXPEDIA (https://www.coexpedia.org/), as shown in Fig. 3D.

The role of $C D C 20$ in GC progression and its expression in GC cells was assessed by RT-qPCR and western blot analysis. The mRNA and protein expression levels of $C D C 20$ were significantly increased in GC cell lines, including MKN-45, MKN-74, AGS, and HGC-27, as compared to the GES-1 cell line (Fig. 3E,F). Moreover, MYBL2 knockdown inhibited protein and mRNA expression of $C D C 20$ (Fig. 3G,H). Consistently with the search results outlined above, the IP assay showed that endogenous CDC20 and MYBL2 formed a complex in HGC-27 cells (Fig. 3I). Collectively, these results suggest that $M Y B L 2$ interacts with $C D C 20$ in vitro.

\section{MYBL2 knockdown inhibits the proliferation and promotes apoptosis of HGC-27 cells through the regulation of CDC20 expression}

To confirm the mechanism by which $M Y B L 2$ regulates GC cell growth, the biological significance of the interaction between $M Y B L 2$ and $C D C 20$ was examined. First, an $\mathrm{Ov}-C D C 20$ plasmid was constructed, and its transfection efficiency was confirmed using RT-qPCR and western blot analysis (Fig. 4A,B). The results from the CCK-8 assay demonstrated that $C D C 20$ overexpression partly abolished the inhibitive effects of $M Y B L 2$ knockdown on HGC-27 cell viability (Fig. 4C) and proliferative markers expressions (Fig. 4D). Consistent with these observations, the results from the colony formation assay showed that $C D C 20$ overexpression reversed the inhibitive effect of $M Y B L 2$ silencing on HGC-27 cell proliferation (Fig. 4E). Furthermore, the results from TUNEL staining showed that the cell apoptosis rate was significantly decreased in HGC-27 cells co-transfected with si-MYBL2 and Ov$C D C 20$ compared to cells transfected with si-MYBL2 alone (Fig. 4F,G). The Bcl-2 expression level in HGC-27 cells from the si-MYBL2+Ov-CDC20 group was higher, while the expression levels of Bax and cleaved caspase-3 was lower, than that in si-MYBL2 group (Fig. 4H,I). These results indicate that $M Y B L 2$ knockdown inhibits the proliferation and promotes the apoptosis of HGC-27 cells through the regulation of $C D C 20$ expression.

\section{Effects of $M Y B L 2$ on the Wnt/ $\beta$-catenin signaling pathway}

To further clarify the underlying mechanisms of $M Y B L 2$ in GC progression, the effects of $M Y B L 2$ on the Wnt/Bcatenin signaling pathway were analyzed. As shown in Fig. 5, MYBL2 knockdown led to a reduction in the expression of $\beta$-catenin, $\mathrm{p}-\mathrm{GSK}-3 \beta$ and mc-Myc, which was reversed by $C D C 20$ overexpression. These results suggest that $M Y B L 2$ knockdown induces inactivation of $\mathrm{Wnt} / \beta$ catenin signaling pathway.

\section{Discussion}

Gastric cancer is a common malignant tumor with a high morbidity and mortality globally. Nowadays, due to risk factors such as changes in diet and lifestyle, the incidence of GC is increasing. ${ }^{19}$ It is a disease that is highly heterogeneous in terms of molecular and cellular phenotype, and is diagnosed histologically through endoscopic biopsy. Endoscopic resection is mainly used for early-stage GC, and surgery is mainly used for advanced GC. ${ }^{1}$ Despite the advances that have been achieved in diagnosis and therapy, the outcome for GC patients remains poor. There are great limitations in the understanding of the etiology of GC, which involves a multifaceted process and complex molecular mechanisms. Hence, it is important to explore promising targets for $\mathrm{GC}$ treatment.

The MYB family of proteins contains numerous subtypes with diverse functions, the majority of which act as transcription factors and have different numbers of MYB domain repeats. This latter feature endows these proteins with the ability to bind DNA. ${ }^{20}$ Rapidly accumulating evidence now suggests that the MYB family regulates the cell cycle to maintain DNA replication, cell survival and proliferation., ${ }^{9,21,22}$ It has also been reported that MYBL2 expression is significantly increased in numerous cancer tissues as compared to adjacent tissue, and is negatively associated with the survival rate of cancer patients. ${ }^{7}$ In addition, $M Y B L 2$ downregulation inhibits the proliferation and DNA replication of gallbladder cancer cells in vitro, ${ }^{23}$ which is consistent with the current findings. Specifically, in the current study, it was observed that MYBL2 is overexpressed in GC cell lines (MKN-45, MKN-74, AGS, and HGC-27) compared to a normal gastric cell line (GES-1). Hence, $M Y B L 2$ may be an oncogene in GC progression. To confirm the role of $M Y B L 2$ in GC progression, $M Y B L 2$ downregulation was induced by the transfection of si$M Y B L 2$, and the survival and apoptosis of HGC-27 cells were detected using CCK-8, colony formation and TUNEL assays. The results suggested that $M Y B L 2$ downregulation inhibits the proliferation and promotes the apoptosis of HGC-27 cells.

In order to explore the underlying mechanisms by which $M Y B L 2$ regulates $\mathrm{GC}$ progression, the molecules that can 
A

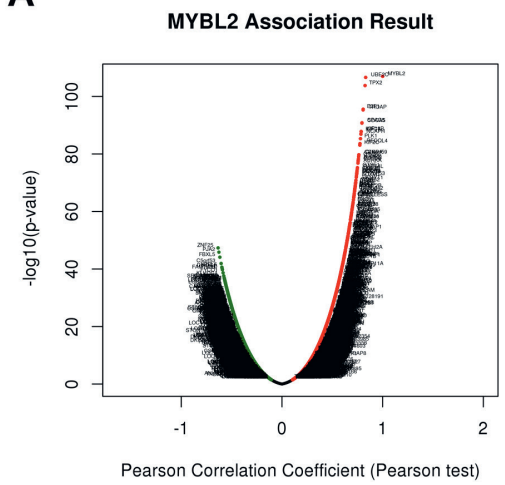

D

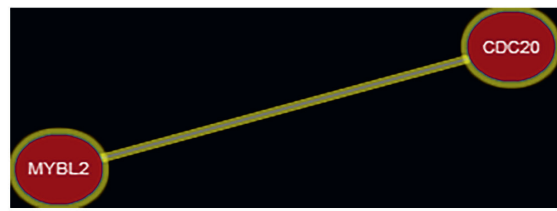

E

CDC20

GAPDH

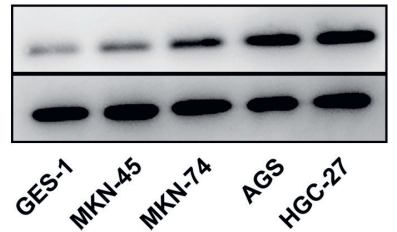

G

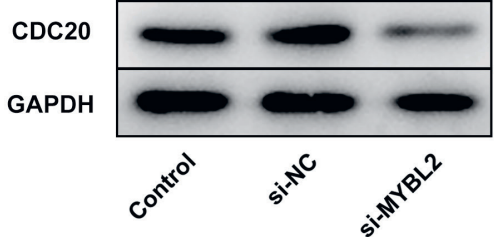

CDC20

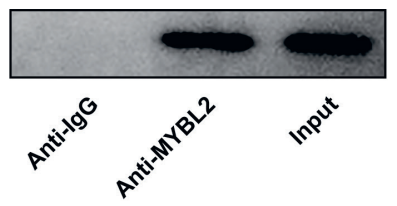

I
C
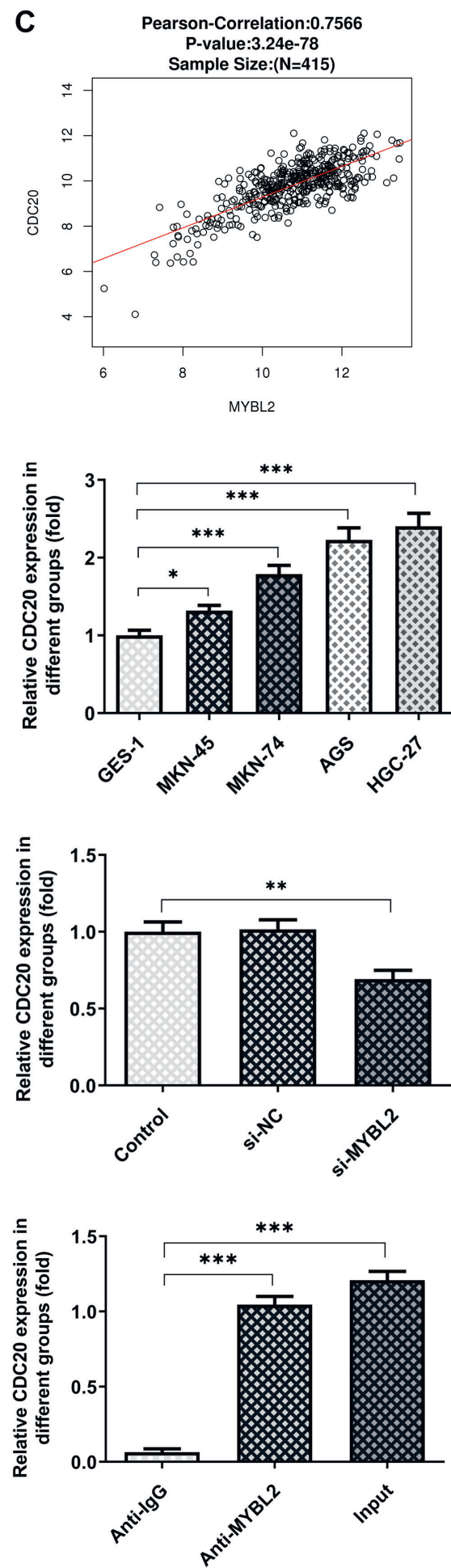

Fig. 3. MYBL2 interacts with CDC20 and requlates its expression. A-C. The correlation between MYBL2 and CDC20 in gastric cancer (GC) was analyzed using the LinkedOmics website; D. The co-expression of MYBL2 and CDC20 in the Gene Expression Omnibus (GEO) database was analyzed using the COEXPEDIA website; $\mathrm{E}$ and $\mathrm{G}$. The CDC20 protein expression was determined with western blot analysis and quantification; $\mathrm{F}$ and $\mathrm{H}$. The CDC20 mRNA level was analyzed with quantitative real-time polymerase chain reaction (RT-qPCR); I. The interaction between MYBL2 and CDC20 was determined using IP. Error bars represent the mean \pm standard error of mean (SEM) from 3 independent experiments

${ }^{*} p<0.05 ;{ }^{* *} p<0.01 ;{ }^{* *} p<0.001$.

interact with this protein were searched on the LinkedOmics and COEXPEDIA websites. The results suggested that MYBL2 expression is positively correlated with $C D C 20$. Consistent with these search results, an IP assay indicated that $M Y B L 2$ interacts with $C D C 20$ in vitro. It has been reported that $C D C 20$ possesses regulatory potential at multiple points of the cell cycle and plays a carcinogenic role in various types of tumor. ${ }^{24}$ For example, 445 breast 
A

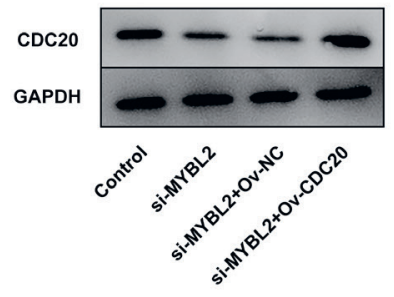

D
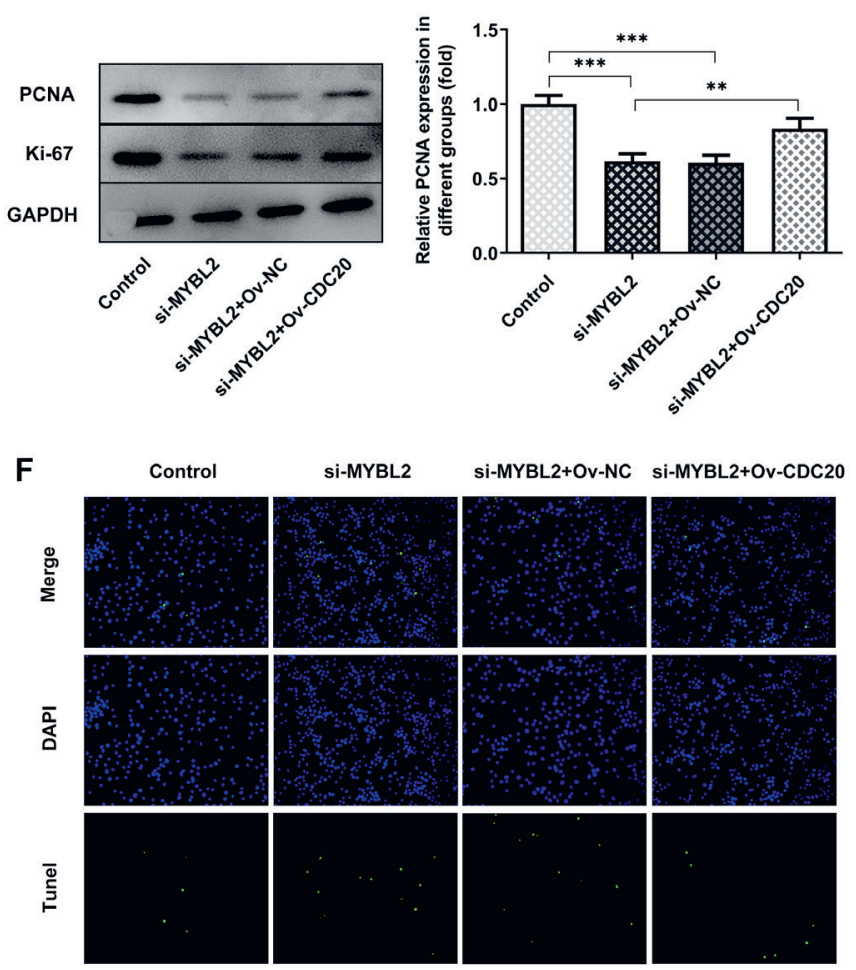

I

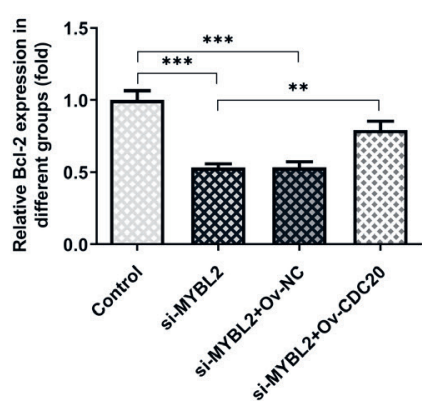

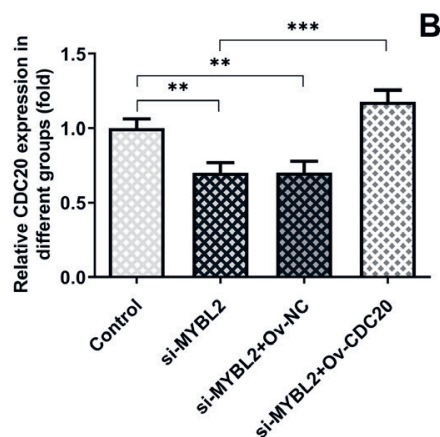

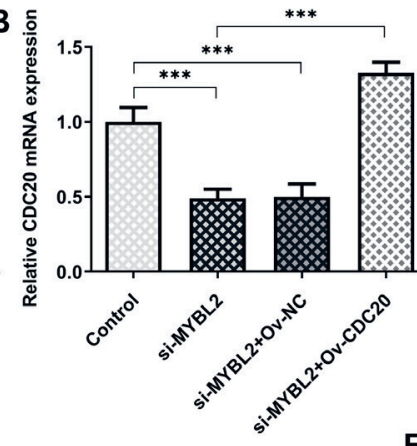

C

口 Control

Di-MYBL2

mi-MYBL2+Ov-NC

a si-MYBL2+Ov-CDC20

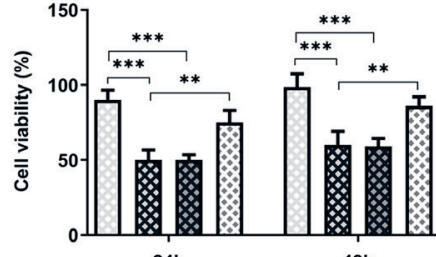

24h

$48 \mathrm{~h}$

E
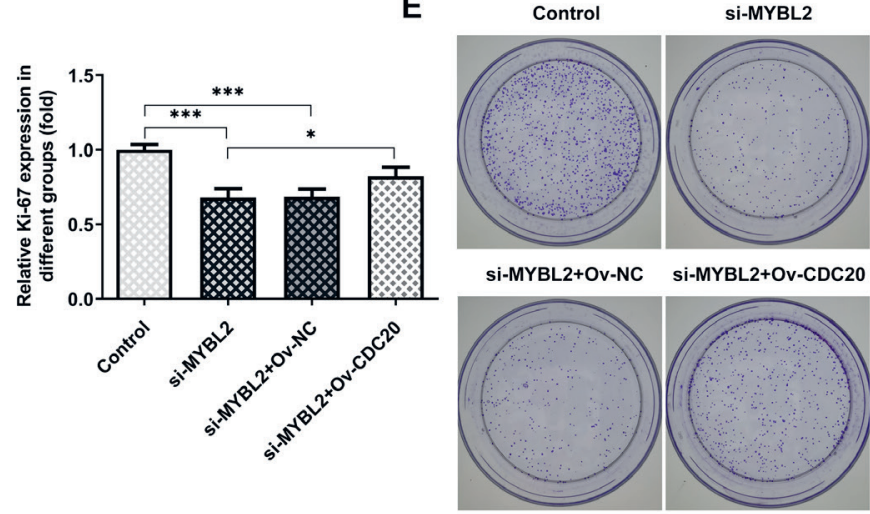

G

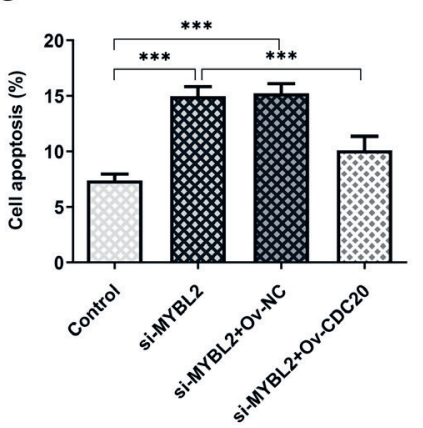

H
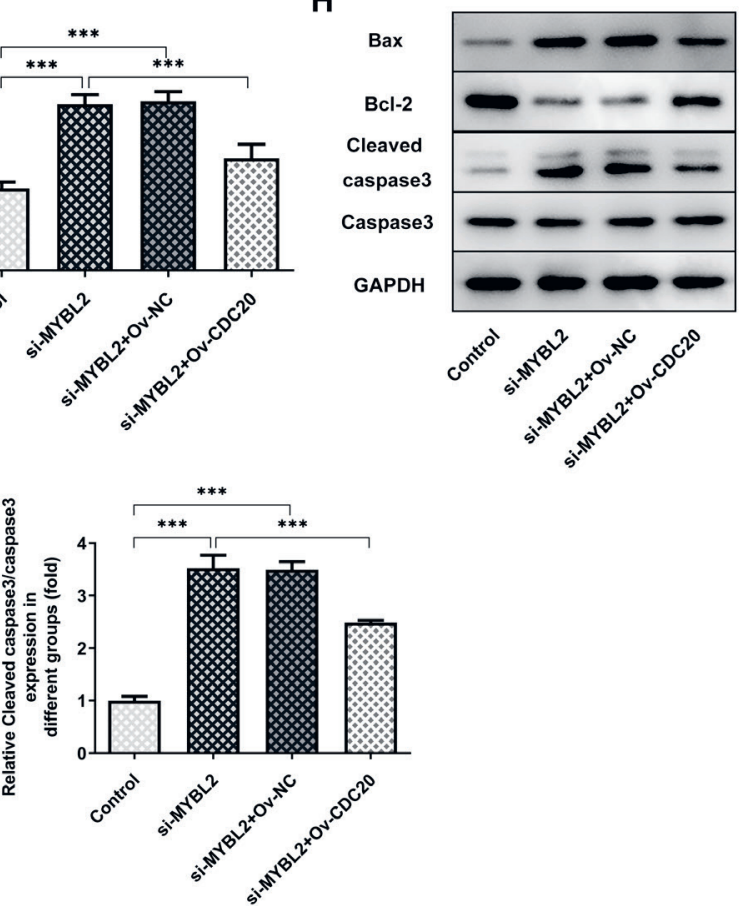

Fig. 4. MYBL2 knockdown inhibits the proliferation and promotes apoptosis of HGC-27 cells via the regulation of CDC20 expression. A. The CDC20 protein expression was determined with western blot analysis and quantification; $\mathrm{B}$. The CDC20 mRNA level was analyzed with quantitative real-time polymerase chain reaction (RT-qPCR); C. HGC-27 cell viability was detected using the Cell Counting Kit-8 (CCK-8); D. The proliferating cell nuclear antigen (PCNA) and Ki-67 protein expressions were determined with western blot analysis and quantification; E. HGC-27 cell proliferation was analyzed with the colony formation assay; F and G. HGC-27 cell apoptosis was determined with TUNEL staining; $\mathrm{H}$ and I. Bax, BCl-2, cleaved caspase-3, and caspase-3, and protein expressions were determined using western blot analysis and quantification. Error bars represent the mean \pm standard error of mean (SEM) from 3 independent experiments

${ }^{* *} p<0.01 ;{ }^{* * *} p<0.001$.

cancer patients were followed up for 20 years to detect the expression of $C D C 20$, which verified that $C D C 20$ is highly expressed in breast cancer patients. In addition, the overexpression of $C D C 20$ was related to the aggressive course of breast cancer. ${ }^{25} \mathrm{CDC} 20$ is also overexpressed in colorectal cancer cell lines and primary cancer tissues. It is worth noting that the expression of $C D C 20$ is relevant to clinical stage, metastasis and short-term overall survival, 

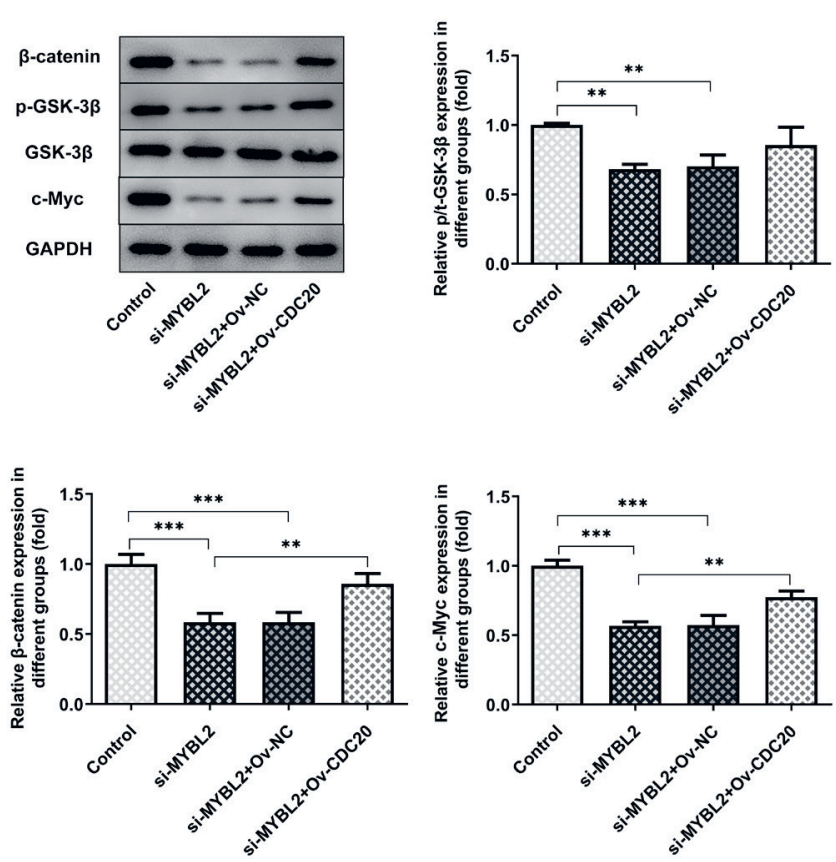

Fig. 5. The effect of MYBL2 on the Wnt/ $\beta$-catenin signaling pathway.

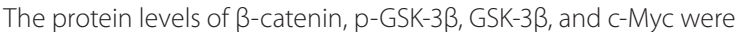
assessed with western blot analysis and quantification. Error bars represent the mean \pm standard error of mean (SEM) from 3 independent experiments

${ }^{* *} \mathrm{p}<0.01 ;{ }^{* * *} \mathrm{p}<0.001$

which indicates that $C D C 20$ can be regarded as an independent prognostic biomarker for human colorectal cancer. ${ }^{26}$ Importantly, a previous study has demonstrated that $C D C 20$ expression is significantly increased in GC tumor tissues compared noncancerous tissues, and its overexpression is closely related to aggressive progression and poor prognosis in GC patients. ${ }^{27} \mathrm{On}$ the basis of the result that MYBL2 silencing depressed $C D C 20$ expression, the biological significance of the interaction between $M Y B L 2$ and $C D C 20$ was subsequently examined. The findings revealed that $C D C 20$ overexpression partly abolished the effect of MYBL2 downregulation on HGC-27 cell proliferation and apoptosis, suggesting that MYBL2 works in synergy with $C D C 20$ to promote the proliferation and inhibit the apoptosis of GC cells.

The Wnt signal transduction cascade is a key driving factor for a variety of tissue stem cells. The Wnt pathway can participate in and can cause a variety of growth-related pathologies and cancers. ${ }^{28}$ In breast, lung and hematopoietic malignancies, activation of the $\mathrm{Wnt} / \beta$-catenin signaling pathway has been found and it mediates tumor recurrence. ${ }^{29,30}$ It has been reported that $C D C 20$ silencing not only suppresses prostate cancer growth, but also enhances chemosensitivity to docetaxel via inhibition of Wnt $/ \beta$-catenin signaling. ${ }^{31}$ In this study, it was found that MYBL2 knockdown induced inactivation of the Wnt/ $\beta$-catenin signaling pathway, while $C D C 20$ upregulation had the opposite effect. Thus, MYBL2, in synergy with
$C D C 20$, promotes the proliferation and inhibits the apoptosis of GC cells, and these effects may involve the Wnt/Bcatenin signaling pathway.

The purpose of this study was to investigate the underlying mechanisms of MYBL2 in the progression of GC. However, the current experiments were carried out in vitro and thus require further investigation in vivo.

\section{Conclusions}

Taken together, the current results indicate that the synergy between MYBL2 and $C D C 20$ induces the proliferation of GC cells and inhibits cell apoptosis, and that these processes may involve the $\mathrm{Wnt} / \beta$-catenin signaling pathway. Thus, $M Y B L 2$, as a promising target, is of great significance for advancing the treatment of GC.

\section{ORCID iDs}

Qianxi Deng (D) https://orcid.org/0000-0002-8901-9191

Linju Wu (D) https://orcid.org/0000-0003-4190-0075

Yiming Li (D) https://orcid.org/0000-0002-0614-8052

Long Zou (D) https://orcid.org/0000-0003-1647-2697

\section{References}

1. Smyth EC, Nilsson M, Grabsch HI, van Grieken NC, Lordick F. Gastric cancer. Lancet. 2020;396(10251):635-648. doi:10.1016/s0140-6736(20) 31288-5

2. Venerito M, Link A, Rokkas T, Malfertheiner P. Gastric cancer: Clinical and epidemiological aspects. Helicobacter. 2016;21(Suppl 1):39-44. doi:10.1111/hel.12339

3. Thrift AP, El-Serag HB. Burden of gastric cancer. Clin Gastroenterol Hepatol. 2020;18(3):534-542. doi:10.1016/j.cgh.2019.07.045

4. Zong $\mathrm{L}$, Abe M, Seto $\mathrm{Y}$, Ji J. The challenge of screening for early gastric cancer in China. Lancet. 2016;388(10060):2606. doi:10.1016/s01406736(16)32226-7

5. Yao J, Zhang H, Liu C, Chen S, Qian R, Zhao K. miR-450b-3p inhibited the proliferation of gastric cancer via regulating KLF7. Cancer Cell Int. 2020;20:47. doi:10.1186/s12935-020-1133-2

6. Jia Y, Gao Y, Li J, Chang Z, Yan J, Qin Y. Prognostic implications of MYBL2 in resected Chinese gastric adenocarcinoma patients. Oncotargets Ther. 2019;12:1129-1135. doi:10.2147/ott.s188820

7. Musa J, Aynaud MM, Mirabeau O, Delattre O, Gruenewald TG. MYBL2 (B-Myb): A central regulator of cell proliferation, cell survival and differentiation involved in tumorigenesis. Cell Death Dis. 2017;8(6): e2895. doi:10.1038/cddis.2017.244

8. Bayley R, Ward C, Garcia P. MYBL2 amplification in breast cancer: Molecular mechanisms and therapeutic potential. Biochim Biophys Acta Rev Cancer. 2020;1874(2):188407. doi:10.1016/j.bbcan.2020.188407

9. Zhang X, Lv QL, Huang YT, Zhang LH, Zhou HH. Akt/FoxM1 signaling pathway-mediated upregulation of MYBL2 promotes progression of human glioma. J Exp Clin Cancer Res. 2017;36(1):105. doi:10.1186/ s13046-017-0573-6

10. Ren F, Wang L, Shen X, et al. MYBL2 is an independent prognostic marker that has tumor-promoting functions in colorectal cancer. Am J Cancer Res. 2015;5(4):1542-1552.

11. Fuster O, Llop M, Dolz S, et al. Adverse prognostic value of MYBL2 overexpression and association with microRNA-30 family in acute myeloid leukemia patients. Leuk Res. 2013;37(12):1690-1696. doi:10. 1016/j.leukres.2013.09.015

12. Li X, Zhang X, Wu CC, et al. The role of MYB proto-oncogene like 2 in tamoxifen resistance in breast cancer. J Mol Histol. 2021;52(1): 21-30. doi:10.1007/s10735-020-09920-6

13. Hartwell LH, Mortimer RK, Culotti J, Culotti M. Genetic control of the cell division cycle in yeast. V. Genetic analysis of cdc mutants. Genetics. 1973;74(2):267-286. PMID:17248617 
14. Li M, York JP, Zhang P. Loss of CDC20 causes a securin-dependent metaphase arrest in two-cell mouse embryos. Mol Cell Biol. 2007; 27(9):3481-3488. doi:10.1128/mcb.02088-06

15. Manchado E, Guillamot M, de Carcer G, et al. Targeting mitotic exit leads to tumor regression in vivo: Modulation by Cdk1, Mastl, and the PP2A/B55a, $\delta$ phosphatase. Cancer Cell. 2010;18(6):641-654. doi:10. 1016/j.ccr.2010.10.028

16. Chang DZ, Ma Y, Ji B, et al. Increased CDC20 expression is associated with pancreatic ductal adenocarcinoma differentiation and progression. J Hematol Oncol. 2012;5:15. doi:10.1186/1756-8722-5-15

17. Wang L, Zhang J, Wan L, Zhou X, Wang Z, Wei W. Targeting CDC20 as a novel cancer therapeutic strategy. Pharmacol Ther. 2015;151: 141-151. doi:10.1016/j.pharmthera.2015.04.002

18. Ma Y, Kong J, Yan G, et al. NOO1 overexpression is associated with poor prognosis in squamous cell carcinoma of the uterine cervix. BMC Cancer. 2014;14:414. doi:10.1186/1471-2407-14-414

19. Yusefi AR, Bagheri Lankarani K, Bastani P, Radinmanesh M, Kavosi Z. Risk factors for gastric cancer: A systematic review. Asian Pac J Cancer Prev. 2018;19(3):591-603. doi:10.22034/apjcp.2018.19.3.591

20. Dubos C, Stracke R, Grotewold E, Weisshaar B, Martin C, Lepiniec L. MYB transcription factors in Arabidopsis. Trends Plant Sci. 2010;15(10): 573-581. doi:10.1016/j.tplants.2010.06.005

21. Qin $\mathrm{H}$, Li Y, Zhang $\mathrm{H}$, et al. Prognostic implications and oncogenic roles of MYBL2 protein expression in esophageal squamous-cell carcinoma. Onco Targets Ther. 2019;12:1917-1927. doi:10.2147/ott.s190145

22. Wei T, Weiler SME, Toth M, et al. YAP-dependent induction of UHMK1 supports nuclear enrichment of the oncogene MYBL2 and proliferation in liver cancer cells. Oncogene. 2019;38(27):5541-5550. doi:10. 1038/s41388-019-0801-y
23. Liang $\mathrm{HB}, \mathrm{Cao} \mathrm{Y}, \mathrm{Ma} \mathrm{O}$, et al. MYBL2 is a potential prognostic marker that promotes cell proliferation in gallbladder cancer. Cell Physiol Biochem. 2017:41(5):2117-2131. doi:10.1159/000475454

24. Chu Z, Zhang X, Li Q, Hu G, Lian CG, Geng S. CDC20 contributes to the development of human cutaneous squamous cell carcinoma through the Wnt/ $\beta$-catenin signaling pathway. Int J Oncol. 2019;54(5): 1534-1544. doi:10.3892/ijo.2019.4727

25. Karra $\mathrm{H}$, Repo $\mathrm{H}$, Ahonen I, et al. CDC20 and securin overexpression predict short-term breast cancer survival. Br J Cancer. 2014;110(12): 2905-2913. doi:10.1038/bjc.2014.252

26. Wu WJ, Hu KS, Wang DS, et al. CDC20 overexpression predicts a poor prognosis for patients with colorectal cancer. J Transl Med. 2013; 11:142. doi:10.1186/1479-5876-11-142

27. Ding ZY, Wu HR, Zhang JM, Huang GR, Ji DD. Expression characteristics of $C D C 20$ in gastric cancer and its correlation with poor prognosis. Int J Clin Exp Pathol. 2014;7(2):722-727. PMID:24551295

28. Nusse R, Clevers $H$. Wnt/ $\beta$-catenin signaling, disease, and emerging therapeutic modalities. Cell. 2017;169(6):985-999. doi:10.1016/j. cell.2017.05.016

29. Krishnamurthy N, Kurzrock R. Targeting the Wnt/beta-catenin pathway in cancer: Update on effectors and inhibitors. Cancer Treat Rev. 2018;62:50-60. doi:10.1016/j.ctrv.2017.11.002

30. Li Y, Jin K, van Pelt GW, et al. c-Myb enhances breast cancer invasion and metastasis through the Wnt/ $\beta$-catenin/axin2 pathway. Cancer Res. 2016;76(11):3364-3375. doi:10.1158/0008-5472.can-15-2302

31. Li K, Mao Y, Lu L, et al. Silencing of CDC20 suppresses metastatic castration-resistant prostate cancer growth and enhances chemosensitivity to docetaxel. Int J Oncol. 2016;49(4):1679-1685. doi:10.3892/ ijo.2016.3671 